\title{
Galactic foregrounds for the CMB
}

\author{
Subir Sarkar* \\ University of Oxford and NBI Copenhagen \\ E-mail: s.sarkar@physics.ox.ac.uk
}

\begin{abstract}
We find imprints of galactic structures viz. the 'radio loops' [1] in the derived maps of the cosmic microwave background, even at microwave frequencies where emission by dust dominates [2]. The emission mechanism may be magnetic dipole radiation from dust grains enriched by metallic iron, or ferrimagnetic molecules [3]. This new foreground is present at high galactic latitudes, in particular in theBICEP2 observational window, and potentially dominates over the expected B-mode polarisation signal due to primordial gravitational waves from inflation .

References

[1] P. Mertsch and S. Sarkar, Loops and spurs: The angular power spectrum of the Galactic synchrotron background, JCAP 1306 (2013) 041; arXiv:1304.1078 [astro-ph.GA].

[2] H. Liu, P. Mertsch and S. Sarkar, Fingerprints of Galactic Loop I on the Cosmic Microwave Background, submitted to Astrophys. J. Lett.; arXiv:1404.1899 [astro-ph.CO].
\end{abstract}

[3] B.T. Draine and A. Lazarian, Magnetic dipole microwave emission from dust grains, Astrophys. J. 512 (1999) 740-754; ;rXiv:astro-ph 9807009.

Frontiers of Fundamental Physics 14 - FFP14,

15-18 July 2014

Aix Marseille University (AMU) Saint-Charles Campus, Marseille

\footnotetext{
${ }^{*}$ Speaker.
} 